\title{
Application Effect of Humanized Nursing in the Perioperative Period of Gastrointestinal Tumor
}

\author{
Wang Ling Yan*, Chen Yan Xia, Tang Chun Yan \\ Department of Gastrointestinal Surgery, Sun Yat-sen University Cancer Center, Guangzhou, China
}

Email address:

wangly@sysucc.org.cn (Wang Ling Yan)

${ }^{*}$ Corresponding author

\section{To cite this article:}

Wang Ling Yan, Chen Yan Xia, Tang Chun Yan. Application Effect of Humanized Nursing in the Perioperative Period of Gastrointestinal Tumor. American Journal of Nursing Science. Vol. 10, No. 4, 2021, pp. 201-204. doi: 10.11648/j.ajns.20211004.12

Received: June 9, 2021; Accepted: July 1, 2021; Published: July 7, 2021

\begin{abstract}
To observe the effect of humanized nursing in the perioperative period of gastrointestinal tumor. A total of 118 cases of gastrointestinal tumor patients treated in our hospital from March 2021 to May 2021 were selected. Patients were randomly assigned to the routine care control group or humanized nursing intervention group according to their willingness. The gastrointestinal function, psychological status, postoperative complications and nursing satisfaction status of the two groups were compared after intervention. The recovery rate of gastrointestinal function was faster in the study group, that is, the time of initial anal exhaust and initial defecation was lower in the study group than in the control group, and the incidence of abdominal distension was lower in the study group $(\mathrm{P}<0.05)$. The psychological status of the study group was significantly better than that of the control group, that is, the scores of all items of the Symptom Checklist-90 (SCL-90) in the study group were lower than those in the control group $(\mathrm{P}<0.05)$. Compared with the control group, the incidence of postoperative complications in the study group was lower and the nursing satisfaction was better $(\mathrm{P}<0.05)$. The findings demonstrate application of humanized nursing for patients in the perioperative period of gastrointestinal tumor not only effectively promote the recovery process of patients, but also alleviate the adverse mood of patients and improve the quality of hospital nursing work, which has clinical application usefulness.
\end{abstract}

Keywords: Gastrointestinal Tumor, Perioperative Period, Humanized Nursing, SCL-90 Score, Effect

\section{Introduction}

As a common malignancy, gastrointestinal tumor has a high incidence rate in digestive system diseases, which mainly affects middle-aged and elderly people [1]. Because of the changes in lifestyle, living standards and quality of life, people will naturally change their dietary structure, and the change of diet structure leads to an upward trend in the incidence of gastrointestinal tumor. Gastrointestinal tumor patients are mainly treated by surgery, but the recovery of patients after surgery is poor, and they may have negative emotions due to the stress stimulation of surgery [2].

Therefore, for the clinical treatment of patients with gastrointestinal tumor in perioperative period, appropriate nursing intervention is very important. The traditional nursing only gives the basic intervention to the patients, but does not pay attention to the psychological state of the patients during the perioperative period, which has a certain adverse effect on the postoperative gastrointestinal function of the patients. Humanized nursing is not only to simply complete the nursing task, but also to think from the perspective of patients, which can play a better intervention effect. Luo, et al [3] found that patients with humanized nursing had higher satisfaction with nursing work after intervention than patients with basic treatment $(93.33 \%$ vs $78.46 \%$ ). Thus we selected part of patients with gastrointestinal tumor in our hospital to study the effect of humanized nursing in the perioperative period.

\section{Materials and Methods}

\subsection{Sample Collection}

A total of 118 cases of gastrointestinal tumor patients 
treated in our hospital from March 2021 to May 2021 were selected. Patients were randomly assigned to the routine care control group or humanized nursing intervention group according to their willingness, 59 cases in each group. There were 34 males and 25 females in the control group, aged from 56 to 76 years old, with an average age of $(63.6 \pm 3.4)$ years old, the course of disease was 59-93 days, the average course was $(71.3 \pm 4.5)$ days. There were 30 males and 29 females in the study group, aged from 54 to 78 years old, with an average age of (64.1 \pm 4.1$)$ years old, the course of disease was 60-96 days, the average course of disease was $(73.2 \pm 4.8)$ days. Inclusion criteria [4]: confirmed gastric or colorectal tumor; Surgical treatment was adopted; Clear consciousness and independent expression; Informed and voluntarily signed a consent to participate in this study. Exclusion criteria [5]: the presence of consciousness disorder; Poor compliance, unable to complete the task of this study according to the doctor's advice; Combined with other malignant tumors. There was no significant difference in baseline data $(\mathrm{P}>0.05)$.

\subsection{Methods of Nursing Intervention}

The control group was given routine nursing intervention, including explaining disease knowledge, reminding patients to change drugs on time, giving patients medication guidance and so on. The study group was given humanized nursing intervention. It mainly includes: (1) psychological intervention. Some patients do not fully understand the operation, and may have anxiety and other negative emotions due to the unknown of the operation during the treatment. These negative emotions will aggravate the pain of patients, which is not conducive to postoperative rehabilitation [6]. Therefore, nurses should communicate with patients patiently, give psychological support and help patients establish confidence. (2) Body position intervention. It is helpful for patients to take correct posture after operation. If the patient has obvious pain, guide the patient to lie down, which can minimize the pressure on the surgical wound and help relieve the pain. Within the acceptable range of patients, allow the patient to take a semi-decubitus position to drain abdominal exudate and pelvic exudate. (3) Diet intervention [7]. According to the patient's postoperative recovery, there should be a period of fasting. After the first anal exhaust, the gastric tube can be removed. After the removal of the gastric tube, the patient needs to eat liquid food for a period of time and the normal diet can be carried out after the gastrointestinal function is restored. (4) Exercise rehabilitation intervention. As long as the patient's physical condition allows, the earlier the rehabilitation exercise starts, the better [8]. However, the intensity and range of rehabilitation exercise also need a gradual cycle, from training respiratory function to physical exercise in bed, and then to standing out of bed step by step [9].

\subsection{Outcome Measures}

Recovery of gastrointestinal function. The gastrointestinal function of the patients was observed in three aspects: the time of first anal exhaust, the time of first defecation and the rate of abdominal distension. The shorter the time of initial exhaust and initial defecation and the lower the rate of abdominal distension indicate that the recovery of gastrointestinal function is better [10].

Psychological assessment. The SCL-90 scale was used to evaluate the psychological status of the patients in 10 aspects, including obsessive-compulsive, psychotic, phobic and depressive. The lower the score of each item, the better the psychological state of patients in this aspect.

Postoperative complications were evaluated in incision infection, pulmonary infection, deep venous thrombosis and adhesive ileus.

Evaluation of nursing satisfaction. Patients were asked to fill in the self-made satisfaction questionnaire to express their satisfaction with nursing work. The full score of the questionnaire was 100. The higher the score, the more satisfied with the nursing work [11].

\subsection{Statistical Analysis}

Spss 20.0 software was used to analyze the patient data. $\chi^{2}$ test and $\mathrm{t}$ test were used to compare the enumeration data and measurement data, respectively. And \% and ( $\bar{x} \pm \mathrm{s})$ were used to express enumeration data and measurement data, respectively. $\mathrm{P}$ values $<0.05$ were considered to indicate statistical significance.

\section{Results}

\subsection{Comparison of the Recovery of Gastrointestinal Function Between the Two Groups}

The recovery speed of gastrointestinal function in the study group was faster. That is, the time of initial anal exhaust and initial defecation in the study group was lower than that in the control group, and the incidence of abdominal distension in the study group was lower $(\mathrm{P}<0.05)$. The detailed data are shown in Table 1.

Table 1. Comparison of recovery of gastrointestinal function between the two groups ( $\overline{x \pm s}$ ).

\begin{tabular}{lllll}
\hline Group & Case & Initial exhaust time of anal (h) & Initial defecation time of anus (h) & Incidence of abdominal distension (\%) \\
\hline Control group & 59 & $64.37 \pm 4.29$ & $73.82 \pm 5.56$ & $8(13.60)$ \\
Study group & 59 & $43.12 \pm 3.67$ & $55.02 \pm 4.91$ & $2(3.40)$ \\
$\mathrm{t} / \chi^{2}$ & $I$ & 28.91 & 19.74 & 3.93 \\
$P$ value & $/$ & 0.000 & 0.000 & 0.0473 \\
\hline
\end{tabular}

\subsection{Comparison of Psychological Status Between the Two Groups}

The psychological status of the study group was significantly better than that of the control group. That is, the scores of each 
item in the SCL-90 scale of the study group were lower than those of the control group $(\mathrm{P}<0.05)$. The detailed data are shown in Table 2.

Table 2. Comparison of psychological status between the two groups (score, $\overline{x \pm s}$ ).

\begin{tabular}{|c|c|c|c|c|c|c|}
\hline Group & Case & Compulsion & Paranoid & Psychoticism & Somatization & Interpersonal relationship \\
\hline Control group & 59 & $2.14 \pm 0.51$ & $1.87 \pm 0.32$ & $1.83 \pm 0.58$ & $1.96 \pm 0.37$ & $2.06 \pm 0.47$ \\
\hline Study group & 59 & $1.67 \pm 0.46$ & $1.46 \pm 0.29$ & $1.57 \pm 0.36$ & $1.43 \pm 0.28$ & $1.68 \pm 0.40$ \\
\hline $\mathrm{t}$ & l & 5.26 & 7.29 & 2.93 & 8.77 & 4.73 \\
\hline$P$ value & / & 0.000 & 0.000 & 0.004 & 0.000 & 0.000 \\
\hline Group & Case & Hostility & Anxiety & Depression & Terror & Other factors \\
\hline Control group & 59 & $2.15 \pm 0.43$ & $1.92 \pm 0.45$ & $1.89 \pm 0.36$ & $1.96 \pm 0.53$ & $2.39 \pm 0.49$ \\
\hline Study group & 59 & $1.49 \pm 0.30$ & $1.62 \pm 0.42$ & $1.54 \pm 0.24$ & $1.66 \pm 0.37$ & $1.89 \pm 0.51$ \\
\hline$P$ value & 1 & 0.000 & 0.000 & 0.000 & 0.001 & 0.000 \\
\hline
\end{tabular}

\subsection{Comparison of Postoperative Complications Between the Two Groups}

Compared with the control group, the incidence of postoperative complications in the study group was lower, and the nursing satisfaction was better $(\mathrm{P}<0.05)$. The detailed data are shown in Table 3.

Table 3. Postoperative complications of the two groups [n (\%)].

\begin{tabular}{|c|c|c|c|c|c|c|}
\hline Group & Case & Incision infection & $\begin{array}{l}\text { Pulmonary } \\
\text { infection }\end{array}$ & $\begin{array}{l}\text { Lower extremities deep } \\
\text { venous thrombosis }\end{array}$ & Adhesive ileus & $\begin{array}{l}\text { Complication } \\
\text { probability }\end{array}$ \\
\hline Control group & 59 & $5(8.5)$ & $2(3.4)$ & $1(1.7)$ & $2(3.4)$ & $10(16.9)$ \\
\hline Study group & 59 & $2(3.4)$ & $0(0.0)$ & $1(1.7)$ & $0(0.0)$ & $3(5.1)$ \\
\hline$\chi^{2}$ & l & & & & & 4.24 \\
\hline$P$ value & l & & & & & 0.0396 \\
\hline
\end{tabular}

\subsection{Comparison of Satisfaction with Prognosis Between the Two Groups}

Compared with the control group, the nursing satisfaction of the study group was better after nursing intervention $(\mathrm{P}<0.05$ ). The detailed data are shown in Table 4.

Table 4. Comparison of prognosis satisfaction between the two groups [n (\%)].

\begin{tabular}{|c|c|c|c|c|c|}
\hline Group & Case & Perfect contentment & Satisfactory & Unsatisfactory & Total satisfaction \\
\hline Control group & 59 & $31(52.5)$ & $16(27.1)$ & $12(20.3)$ & $48(80.0)$ \\
\hline Study group & 59 & $37(62.7)$ & $18(30.5)$ & $4(6.8)$ & $55(93.2)$ \\
\hline$\chi^{2}$ & l & & & & 4.47 \\
\hline$P$ value & 1 & & & & 0.345 \\
\hline
\end{tabular}

\section{Discussion}

As a common malignant tumor of digestive tract, the current treatment of gastrointestinal tumor is comprehensive treatment, with surgical resection as the main way. Although most of the patients have good surgical effect, the exogenous stimulation brought by the operation will make the patients' psychology and mood adversely affected, which has a negative superimposed effect on the surgical effect [12]. Therefore, it is very important to give effective nursing intervention to patients after operation to ensure the curative effect of operation. Humanized nursing is a common nursing intervention measure in recent years, which focuses on regulating the psychological condition and adjusting the physical condition of patients.

In this study, the SCL-90 scale items in the study group were lower than those in the control group $(P<0.05)$. It was proved that humanized nursing intervention can provide effective psychological support to patients and help the establishment of positive emotions. The humanized nursing intervention can effectively eliminate negative emotions and make patients more active in cooperation with the treatment [13]. Good mood can also positively affect the recovery of patients. In this study, compared with the control group, the study group not only had the first exhaust (43.12 $\pm 3.67 \mathrm{~h}$ vs $64.37 \pm 4.29 \mathrm{~h}$ ) and the first defecation time was shorter (55. $02 \pm 4.91 \mathrm{~h}$ vs $73.82 \pm 5.56 \mathrm{~h}$ ), and the incidence of abdominal distension was lower (3.4\% in the study group and $13.6 \%$ in the control group) $(\mathrm{P}<0.05)$, which proved this. Moreover, in terms of the satisfaction of nursing work, the study group patients' satisfaction was as high as $93.2 \%$, while the control group's satisfaction was $80.0 \%$, the difference of satisfaction between the two groups was significant $(\mathrm{P}<0.05)$. This may be because humanized nursing not only gives effective care to patients during hospitalization, but also integrates the nursing work after discharge into the scope of humanized nursing intervention [14]. The nursing staff will issue life instruction manual to the discharged patients, leave the contact information of the patients, follow up the patients regularly, guide the lifestyle and diet after discharge, so as to ensure that the patients also have a good recovery effect in the long term, 
so that the satisfaction of the patients will be improved naturally [15].

To sum up, the application of humanized nursing for patients in the perioperative period of gastrointestinal tumor can not only effectively promote the rehabilitation process of patients, but also alleviate the bad mood of patients, improve the quality of hospital nursing work, which has clinical application usefulness.

\section{References}

[1] JH Wang. Analysis of application effect of accelerated rehabilitation surgery nursing in perioperative period of colon cancer. Practical Journal of cardio cerebrovascular diseases. 2020, 28 (S1): 233-235.

[2] MH Fu, LN Huang, MJ Cai, et al. Application study on medical pillow combined with ear acupoint sticking in relieving anxiety and depression in perioperative colon cancer patients. Journal of Guangzhou University of traditional Chinese Medicine. 2020, 37 (06): 1076-1081.

[3] HJ Luo, SJ Zeng, YP Zeng. Effect of humanized nursing intervention on elderly patients with colon cancer. Shenzhen Journal of integrated traditional Chinese and Western Medicine. 2017, 027 (002): 174-175.

[4] YN Wang, J Zhang. To observe the effect of humanized nursing in prenatal care of 43 high-risk pregnant women. Clinical Laboratory Journal. 2019, 8 (03): 149-150.

[5] N Zhang. Application effect observation of high quality nursing in cerebral thrombosis nursing. Shanxi Medical Journal. 2020, 49 (05): 621-623.

[6] HH Liu, XL Mao, DW Li. Effect of humanized nursing in operating room on postoperative rehabilitation of patients undergoing laparoscopic radical resection of colon cancer. Chinese Journal of Clinical Oncology and Rehabilitation. 2020, v. 27 (04): 89-91.
[7] XY Qi, YJ Li, XH Zhang. Effect of multidisciplinary consultation model in patients undergoing colon cancer surgery. Oncology Progress. 2020, v. 18 (06): 105-108.

[8] GM Chen, CG Zhang, GH Gan. Nursing application of rapid rehabilitation concept in laparoscopic surgery for colon cancer. Journal of Chengdu Medical College. 2020, 015 (003): 400-404.

[9] ZY H, Y Liu, HL Mei. Analysis of the application of the concept of rapid rehabilitation surgery in patients with colon cancer complicated with ileus. Journal of Clinical Surgery. 2019, 027 (005): 404-407.

[10] LH Cao, Y Wu. Application value of humanized nursing in perioperative nursing of breast cancer patients. Modern Journal of Integrated Traditional Chinese and Western Medicine. 2019, 028 (017): 1929-1932.

[11] ZJ Jiang, L Guo, H Xue, et al. Application value of humanized nursing in perioperative nursing of patients with cervical cancer. Modern Journal of Integrated Traditional Chinese and Western Medicine. 2019 (23): 2604-2607.

[12] JR Guan, R Liu. Effect of perioperative three-step nursing intervention on operative tolerance and complications in patients with colon cancer. 2020, v. 29 (24): 100-103.

[13] Jun Li, XL Li, Y Yuan, et al. Discussion and exploration of neoadjuvant and adjuvant therapy in perioperative colon cancer. Chinese Journal of Gastrointestinal Surgery. 2019, 22 (4): 329-335.

[14] ZW Zhao, XN Xi, YL Liu, et al. Application of the concept of accelerated rehabilitation surgery in elderly patients with right colon cancer. Chinese Journal of Bases and Clinics in General Surgery. 2019, 026 (001): 77-81.

[15] LF Cheng, YY Cheng, CQ Zhu. Application of rapid rehabilitation concept in nursing care of patients with colon cancer undergoing laparoscopic surgery and its impact on inflammatory reaction. Guangdong Medical Journal. 2020, 41 (07): 742-745. 